\title{
Diseño de una Red de logística Inversa para recolectar Aceite Vehicular Usado en la ciudad de Pereira implementando CVRP
}

\author{
Design of a Reverse Logistics Network to recover Oil Used in Pereira \\ implementing CVRP
}

\author{
Juliana Castañeda Jiménez, Jaime Andrés Cardona Arias \\ Ingeniería Industrial, Universidad Tecnológica de Pereira, Pereira, Colombia \\ jcastaneda@utp.edu.co \\ jaiandcardona@utp.edu.co
}

\begin{abstract}
Resumen- La problemática ambiental generada por la mala disposición de aceite vehicular usado en Colombia ha permitido que en algunas de las principales ciudades se hayan creado empresas destinadas a la recolección y correcta disposición del desecho, pero eso no garantiza una cubertura total de las mismas. La ciudad de Pereira cuenta con una alta cifra de agentes generadores (Lubricentros, Servitecas, Talleres de mecánica, etc.), lo cual hace necesaria la creación de un esquema para la recuperación y adecuada disposición del residuo con el fin de beneficiar al medio ambiente y los habitantes de la ciudad.
\end{abstract}

Este documento presenta la creación de una red de logística inversa asociada a la recolección de aceite vehicular usado (AU) en la ciudad de Pereira. Para la construcción del modelo se ubicaron los puntos generadores del residuo, las distancias entre cada uno de los nodos, y los costos logísticos variables evaluando dos tipos de camiones cisterna. La situación fue modelada como un problema de ruteamiento de vehículos con restricción de capacidad CVRP (Capacited Vehicle Routing Problem), el cual fue solucionado implementando una heurística de dos fases que consiste en Rutear primero y Agrupar después o route-firts and cluster-second. La primera instancia se resuelve usando el Algoritmo de Ahorro y la segunda usando el Algoritmo de Agrupamiento (Clustering), ambos programados en Matlab. La solución determina las sub-rutas que integran la red de logística inversa con el respectivo orden en que cada vehículo debe visitar los diferentes puntos generadores de aceite vehicular usado.

Palabras clave - aceite vehicular usado, agentes o puntos generadores de aceite usado, logística inversa, capacited vehicle routing problem, clustering, costos operativos, heurística del ahorro.

Abstract- The environmental problem generated by the poor used oil discard mechanisms at the present time in Colombia has enable the establishment of business focused on the proper collection and disposal of waste in some of Colombia's major cities; however, this mechanisms still do no guarantee that these companies provide full coverage. The city of Pereira has a high number of oil generating agents (such as Lubrication Centers,
Service Centers, Repair shops, among others), which requires the creation of a proper oil waste recovery and disposal system in order to benefit the environment and the citizens.

This paper presents the creation of a reverse logistics network associated with the proper used oil collection in the city of Pereira. To build the model it was necessary to identify the residue generators points, the distances between each of the nodes and the logistics variable costs evaluating two types of tanker trucks. The situation was modeled as a Capacited Vehicle Routing Problem (CVRP), which was solved by implementing the route-first cluster-second principle in vehicle routing, a two phase heuristic. The first phase is solved by using the Savings Algorithm, while the second phase is solved by using the Clustering Algorithm. Further on, both algorithms are programmed in MATLAB, where the solution determines the sub-routes integrating the reverse logistics network with the order in which each vehicle should visit the different used oil generating points.

Key Word - capacited vehicle routing problem, clustering, oil generating agents or points, operating costs, reverse logistics, savings heuristic, used oil.

\section{INTRODUCCIÓN}

En la actualidad, el tema ambiental ha dejado de preocuparse únicamente por el agotamiento y la escasez de recursos, y se ha interesado en la sostenibilidad, que consiste en utilizar como materia prima no solo recursos naturales, sino también aquellos productos fuera de uso, con la finalidad de reintegrarlos a la cadena de valor.

Las empresas actuales están prestando mayor atención a la disposición final de los residuos peligrosos con el fin de evitar daños ambientales, económicos y sanciones legales. Para ello, se han desarrollado a lo largo del mundo miles de sistemas logísticos que permiten la recuperación de este tipo de 
desechos (llantas usadas, aceite usado, baterías, filtros, etc.) y su posterior reintegración a un proceso productivo.

A diferencia de esas redes de logística inversa, este trabajo tiene como novedad la implementación de técnicas heurísticas para el diseño de una red dedicada a la recolección de aceite vehicular usado que se origina en la ciudad de Pereira, que garantiza la optimización de los costos de transporte de forma tal que la recolección del desecho resulte viable económica y ambientalmente.

Según un informe técnico de la empresa productora de lubricantes para automóviles Gulf, en Colombia salieron al mercado en el 2012, aproximadamente 45 millones de galones de aceite lubricante para motor. Después de finalizada la vida útil del aceite, un alto porcentaje no se dispone adecuadamente y el desecho termina contaminando fuentes hídricas, el ambiente y también es utilizado para falsificar lubricantes. Este último punto afecta económicamente a los productores de lubricantes (Terpel, Castrol, Chevron, etc.) ya que el $20 \%$ del lubricante de motor que se encuentra en el mercado es falso. En Colombia, la cantidad de aceite usado que no se recolecta es altísima y escandalosa, según la UPME (Unidad de Planeación Minero Energética), en el 2008 la FAU (Fondo de Aceites Usados), entidad encargada de la recolección y gestión del residuo, tan solo recolecto el 34,1\% del total de aceite usado estimado que se generó en el país.

Por las razones anteriores, se considera necesario encontrar soluciones o metodologías que viabilicen la recolección del residuo en el país, pero inicialmente con este trabajo en la ciudad de Pereira.

El problema planteado se identificó como un CVRP (Capacited Vechicle Routing Problem) por la restricción de capacidad presente en los camiones utilizados, y para solucionarlo se implementa la heurística del ahorro en una primera instancia y posteriormente se realizaron agrupamientos (clusters) que cumplieran con la restricción de capacidad de los vehículos. Obteniendo así, una red diseñada para la recuperación del aceite usado y la disminución del desecho que se dispone inadecuadamente en la ciudad.

Los altos costos de transporte son los que no permiten que la gestión de aceites usados llegue a muchos puntos de acopio, pero asignando las rutas a los vehículos con la implementación del VRP (Vehicle Routing Problem), se pretende optimizar los costos del recorrido y las distancias, haciendo que la red de logística inversa que se propone para la gestión de los residuos fortalezca el sistema de recolección.

La ruta generada como solución, iniciara el recorrido en un punto donde se almacenará todo el lubricante usado recolectado por los vehículos que recorren la red, allí mismo finalizaran el recorrido. El modelo matemático tendrá en cuenta algunas variables, como las distancias entere puntos, el número de vehículos recolectores, la capacidad de estos, etc.
Generando así una alternativa que optimice la recolección del aceite vehicular usado en la ciudad de Pereira y al mismo tiempo contribuya a la disminución de la manipulación inadecuada de este desecho.

\section{MARCO TEÓRICO}

\section{A. Logística inversa}

Inicialmente, en la década de los 70's apareció la idea de recolectar desechos a partir de la preocupación por el aumento de los residuos generados a causa de la producción en masa en los países industrializados. Fueron estas complejas necesidades las que hicieron que la Logística Inversa apareciera. En los últimos años se ha presentado un especial interés en este tipo de procesos, donde se recolectan los productos que han terminado su vida útil o que han sido desechados por los consumidores, pero aún es posible recuperarlos, a través de la reutilización, el reciclaje o la refabricación. Las tres actividades mencionadas anteriormente, son las que generan mayor valor en la recuperación de valor de los residuos [1].

Los principales motivos que llevan a que se origine un flujo de logística inversa son: motivos económicos, de marketing, legales, post-consumo o de protección de activos de la empresa. [2]

Después de recolectar el producto existen diferentes actividades según Girdhar, Dyckhoff y Langevin a realizar para transformar los productos recuperados, las cuales son: el reúso, la reventa o redistribución, elreprocesamiento, el reciclaje y como último recurso la eliminación.

El proceso de la logística inversa está conformado por 6 actividades principales:

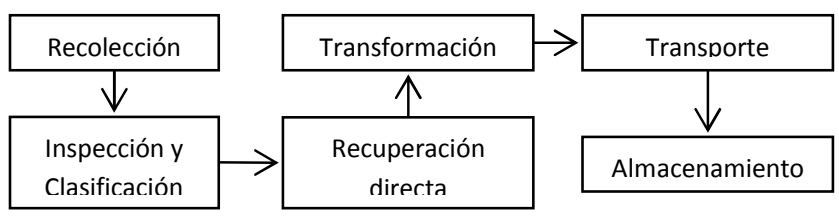

Figura 1. Proceso logístico inversa

A través de este trabajo se impactará el proceso de logística inversa directamente en las actividades de transporte. Se diseñarán rutas con el fin de optimizar los costos y aprovechar adecuadamente la capacidad de los camiones que transportarán el AU. [3]

\section{B. Problema de Enrutamiento de Vehículos (VRP)}

Debido a la importancia que actualmente tiene la recolección y reutilización de residuos contaminantes en el mundo, el problema de enrutamiento de vehículos VRP (Vehicle Routing Problem), se ha ido desarrollando con el paso del tiempo e 
implementando en diferentes sectores económicos, porque es considerado como un problema de optimización combinatorial que contribuye al desarrollo de una región o país, ya que contar con una buena red de transporte y/o comunicación facilita las relaciones comerciales entre las diferentes empresas. Este tipo de problema presenta unas características principales que permite catalogarlo como un VRP, las cuales son:

- La demanda de los clientes debe ser conocida, excepto aquellas variantes del problema en que se cuenta con demandas estocásticas.

- Las visitas a los clientes se debe realizar con el costo mínimo, por lo que resulta necesario optimizar el recorrido de los vehículos, el cual puede o no iniciar y finalizar en el depósito y/o almacén.

- Los clientes, comúnmente son visitados una vez, lo que obliga a tener vehículos de mayor capacidad a la demanda del cliente.

Y también presenta unas variantes que en general presenta los siguientes aspectos en común:

- Tener unos clientes con una demanda que debe ser satisfecha por un solo vehículo. En la mayoría de los problemas, la demanda corresponde a un bien que tiene un volumen asignado dentro del vehículo, también puede entenderse como un periodo de tiempo en el que se presta un servicio al cliente, y en este solo debe ser visitado.

- Contar con depósito y/o almacén donde se encuentran los bienes y los vehículos, por lo general la mayoría de los problemas inician y finalizan en este punto, presentando en algunas ocasiones restricciones de tiempo para la carga o descarga de los bienes dentro del depósito.

- Poseer una flota de vehículos, para el transporte de bienes y/o servicios, con una capacidad limitada por volumen, peso, número o tiempo de visita máxima a los clientes. En casi todos los problemas planteados hasta el momento, es común encontrar que un vehículo de la flota realice solo una ruta, pero actualmente se han planteado otros en los que un vehículo realiza varias rutas.

Las variantes del VRP se encuentran directamente relacionadas con las características presentes en el contexto del problema, es decir las restricciones y/o características de las variables que permiten plantear una aproximación a los problemas reales, que normalmente tienen variaciones en su organización como son el número de depósitos y vehículos, el tipo de demanda de los clientes, que puede estar relacionado con un tiempo límite de visita (ventanas de tiempo) o requiera de varios depósitos, las restricciones de los vehículos en cuanto a capacidad, tiempo de carga y descarga de bienes o del recorrido total, y otras variables relacionadas con el tiempo que la organización vial de los lugares pertenecientes al problema, como el número de paradas obligatorias o el tráfico vehicular, requiere para realizar el recorrido.

Los diferentes casos de VRP que se pueden encontrar en logística inversa son:

\section{VRP con Capacidades (CVRP)}

El VRP con restricción de capacidad es un problema de optimización NP-Hard que cuenta con uno a varios vehículos con una capacidad máxima de carga igual para todos los de la flota. Las cantidades demandas por los clientes se determinan previamente y se dividen entre el número de vehículos sin exceder la capacidad o fraccionar la demanda de los clientes. El problema asigna las cargas a cada vehículo empleando las características de un problema de embalaje BPP (Bin Packig Problem) y la asignación de la ruta se plantea como un TSP con el objetivo de minimizar los costos de transporte. Las características del CVRP son:

- Cada ruta asignada a un vehículo inicia y finaliza en el depósito.

- Cada cliente pertenece a una sola ruta.

- La demanda de los clientes es menor a la capacidad de los vehículos.

- La suma de las demandas de los clientes visitados en una ruta no debe ser mayor que la capacidad total del vehículo.

\section{- VRP con Múltiples Depósitos (MDVRP)}

El VRP con múltiples depósitos es un problema con varios depósitos ubicados en diferentes puntos de una zona geográfica determinada, donde cada uno posee una flota de vehículos usados para satisfacer la demanda de todos los clientes asignados y no es posible separar los clientes por grupos, ya que si el problema permite agruparlos de forma que queden cerca de los depósitos es posible resolverlo como un VRP. [4]

\section{- VRP con Flota Heterogénea (HFVRP)}

En este problema, los vehículos que conforman la flota son heterogéneos, es decir, poseen diferentes capacidades de almacenamiento o formas de almacenar la carga. La capacidad y los tipos de camiones dependen de los parámetros y las características de la demanda que cubre cada vehículo a través de la ruta recorrida. [5]

\section{- $\quad$ VRP con Ventanas de Tiempo (VRPTW)}

Este VRP contiene una restricción donde se considera un espacio de tiempo límite conocido para atender a cada cliente, periodo en el cual el cliente está disponible para recibir la visita del vehículo. Este problema tiene otras generalidades donde existen ventanas de tiempo para la llegada a los clientes, el recorrido hasta los clientes, la llegada de la carga al depósito y el servicio activo de vehículos o conductores. [6] 
- VRP con Recolección y Entrega (VRPPD)

Normalmente se conoce en inglés como Vehicle Routing Problem with Pick-Up and Delivery y en esta variante, además de hacer las entregas a los clientes, al mismo tiempo se recogen los productos que son retornados al fabricante. En este problema se debe hacer especial énfasis en la capacidad del vehículo, debido a que constantemente están entrando y saliendo productos del camión y se debe garantizar que la carga de productos que se recogerá durante el recorrido no supere la capacidad del camión.

\section{- VRP Estocástico (SVRP)}

En este caso algunas variables del problema son consideradas aleatorias y varían en el tiempo. Una de las tres variaciones conocidas, es cuando la demanda de los clientes es estocástica y se determina a través de una función de probabilidad. La segunda, cuando la variable aleatoria son los clientes y su cantidad varía en función del tiempo. Por último, cuando los tiempos del recorrido entre clientes son variables estocásticas.

\section{Metodologías de solución}

Actualmente para las empresas que trabajan con la recolección y/o entrega de bienes y servicios, la reducción de tiempos y costos logísticos juega un papel importante dentro de sus utilidades y la relación con los clientes, convirtiendo el VRP en una herramienta útil para modelar los problemas presentados, y obtener soluciones optimas que cumplan con los objetivos de la empresa y al mismo tiempo permitiendo que sea más competitiva en el mercado.

Los problemas de VRP hacen parte de muchas investigaciones realizadas en los últimos años gracias a las soluciones optimas que se han ido desarrollando con la implementación de diferentes técnicas que van de acuerdo a la complejidad del VRP, que matemáticamente se define del tipo NP (nondeterministic polynomial time). Por ejemplo, los casos en los que el número de nodos no es muy grande es común emplear técnicas exactas, pero si al contrario el problema consta de muchos nodos (como en este caso) y el modelo es complejo, se deben aplicar técnicas no exactas que dan una buena solución cercana a la óptima y disminuyen los tiempos empleados para determinarla, como son las heurísticas y metaheurísticas.

Estas técnicas con el pasar de los días se han convertido en herramientas de fácil manejo gracias al constante desarrollo computacional que ha permitido mejorar los tiempos de ejecución y navegar fácilmente por los sistemas de información geográfica. Los algoritmos utilizados para la solución del VRP se muestran a continuación:

\section{Técnicas Exactas}

Las técnicas exactas son algoritmos que siempre ofrecen una solución óptima, pero que no pueden ser implementados para resolver problemas complejos o con muchas variables por su complejidad en tiempo computacional. Generalmente estas técnicas se emplean para resolver problemas con pocos clientes, y son desarrollados en su mayoría para fines académicos. Los métodos de solución más comunes para los VRP son las heurísticas y meta-heurísticas, ya que los métodos exactos en algunos casos resultan ser inadecuados por su nivel de complejidad y número de clientes [7].

Pero lo anterior no significa que no se haya empleado ningún método exacto para resolver CVRP, por el contrario, existen dos técnicas que se destacan en la solución de este problema, el Método de ramificación y acotamiento [8] y Método de ramificación y corte [9].

\section{Heurísticas}

Las heurísticas son consideradas como técnicas simples que realizan una exploración limitada del espacio de búsqueda y ofrecen una solución factible en un periodo de tiempo aceptable. Las heurísticas utilizadas en la solución de CVRP, por lo general, se clasifican en métodos constructivos, de dos fases, y de mejoramiento, a continuación se explicaran los dos primeros métodos en profundidad y el ultimo solo se mencionará.

\section{- Método de ahorro (Clarke Y Wright 1964)}

Ha sido unos de los algoritmos más implementados para resolver VRP en general, el cual consiste en realizar una exploración limitada del espacio de búsqueda y dar una solución de calidad aceptable en un tiempo moderado. El algoritmo se desarrolla partiendo de una solución con dos rutas $(0, \ldots, i, \ldots, 0)$ y $(0, \ldots, j, \ldots, 0)$, las cuales pueden ser combinadas generando así una sola ruta $(0, \ldots, i, j, \ldots, 0)$ como

se

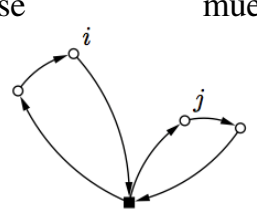
muestra

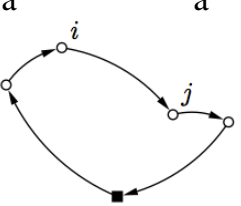

Figura 2. Dos rutas antes y después de ser unidas

El ahorro en distancia se encuentra representado por la siguiente ecuación:

$$
S_{i j}=C_{i 0}+C_{o j}-C_{i j}
$$

Al unir las rutas, los $\operatorname{arcos}(i, 0)$ y $(0, j)$ desaparecen y se agrega el arco $(i, j)$. El algoritmo parte de una solución inicial y realiza las uniones que mayor ahorro generen siempre y cuando cumpla con las restricciones planteadas en el problema. Para usar este método es necesario conocer los costos o las distancias que existen entre los diferentes nodos, o sea el costo de cada trayecto.

\section{- $\quad$ Método de Inserción}


Es un método que consisten en construir la solución insertando clientes que no se han incluido en ninguna de las rutas existentes, y se puede hacer de forma secuencial, o sea que los clientes se van insertando a la última ruta creada siempre y cuando no se incumpla ninguna restricción, pero esto puede generar la inserción de clientes que están muy dispersos aumentado el costo total de las ultimas rutas, por lo que se ha desarrollado otra forma, la paralela que permite insertar el cliente en cualquier ruta existente en la solución. [10]

Los algoritmos de inserción comienzan seleccionando un ciclo inicial (sub-ruta) con $\mathrm{n}$ nodos, posteriormente los $\mathrm{j}$ son seleccionados e insertados en la ruta, de acuerdo a una o varias restricciones, donde la longitud del ciclo no se incremente. Los criterios más utilizados en la solución de CVRP han sido:

Inserción más cercana (El Vecino Más Cercano): Seleccionar el vértice j más cercano al ciclo.

Inserción más lejana: Seleccionar el vértice j más lejano al ciclo.

Inserción de menor costo: Seleccionar el vértice $\mathrm{j}$ que será insertado con el menor incremento del coste. Inserción aleatoria: Seleccionar el vértice j al azar.

\subsection{Heurísticas de dos fases}

Las heurísticas de dos fases permiten construir soluciones factibles que normalmente no resultan óptimas, para ello dividen el problema en dos fases, la primera para el agrupamiento de los nodos y la segunda para la asignación de las rutas a los vehículos. En estas heurísticas sobresalen dos métodos que se explicaran a continuación, y que implementan un gran número de algoritmos para llegar a la solución.

\section{- Método De Rutear Primero Y Asignar Después (Routing First Clustering Second)}

El planteamiento básico sobre este método puede ser adaptado a cualquier heurística y se desarrolla en dos fases. En la primera se usa un algoritmo que genere una ruta $\mathrm{R}$ donde se incluyen todos los nodos como si fueran a ser visitados por un solo vehículo sin tener en cuenta las restricciones de capacidad del problema. Esta ruta resulta ser la solución óptima que en la segunda fase va a ser agrupada (clustering) de forma que la capacidad del vehículo sea optimizada y no se exceda. [11]

Para la primera fase se puede implementar cualquier método de solución que sea apropiado para la construcción de la ruta $R=(0, i, \ldots, j, 0)$, como los mencionados anteriormente, el método de ahorro y de inserción. Para la segunda fase que es la de asignación, se debe dividir $\mathrm{R}$ de forma que cumpla con la restricción de capacidad de los camiones, se puede utilizar el método de partición optima planteado por Beasly el cual consiste en dividir la ruta $\mathrm{R}$ en sub-rutas factibles, planteando el problema como el de hallar la ruta más corta.
Este problema consiste en una red en la que cada arco $(i, j)$ tiene asociado una variable (demanda, costo, distancia o tiempo) que significa el valor que toma desde el nodo $i$ hasta el nodo $j$. Para el caso de asignación en la solución de un CVRP, se debe realizar la sumatoria de las demandas de forma que no se exceda la capacidad del camión, y así ir generando las sub-rutas que en algunos casos se construyen de la siguiente manera $(0, i+1, \ldots, j, 0)$. Este problema también ha sido exitosamente solucionado implementado el algoritmo de Dijkstra, el cual ofrece una solución rápida y eficiente. Teniendo en cuenta el planteamiento original del método, se sabe que en el nodo $i+1$ que inicia la ruta, siempre va a seguir el orden asignado en la primera fase, y solo se puede modificar para obtener una mejor ruta, variando el orden de los clientes [12].

\section{Metaheurísticas}

Las técnicas metaheurísticas permiten realizar una exploración más profunda del espacio de soluciones, por lo que las soluciones arrojadas resultan ser de mejor calidad que las de las heurísticas, pero con un tiempo de ejecución mayor que sigue siendo inferior al que utilizan las técnicas exactas. Los métodos metaheurísticos se dividen en:

\subsection{Métodos constructivos}

Se desarrollan de igual forma que los que pertenecen a las heurísticas, es decir, parten de una solución vacía y luego empiezan a introducir nodos a las rutas evaluándolos de acuerdo a las restricciones y la función objetivo. Entre los algoritmos más usados en este método se encuentra el de GRASP.

\section{- Algorithm de GRASP (Greedy Randomized Adaptive Search Procedure)}

En español es conocido como "Procedimiento de Búsqueda Voraz Aleatorio y Adaptativo", fue desarrollado por fue desarrollado por T.A. Feo and M.G.C. Resendeen 1995. La implementación del algoritmo se divide en cada iteración en dos fases, una constructiva y otra de mejoramiento.

En la fase constructiva, por lo general emplea heurísticas constructivas para obtener una solución inicial, que en la segunda fase va a ser mejorada con un algoritmo de búsqueda local. El algoritmo se caracteriza por seleccionar el nodo a incluir que mejor resultado arroje y actualizar la solución final con el valor del nuevo nodo [13].

\subsection{Métodos evolutivos}

Son métodos que arrojan en una primera instancia soluciones completas, basadas en las variabilidades del VRP, y luego las combina para generar otro grupo de soluciones, hasta llegar a una solución factible. Los algoritmos más utilizados en la 
solución de CVRP son el algoritmo genético y de búsqueda dispersa.

\section{- Algoritmo genético}

Es un algoritmo que se basa en la teoría de la evolución de especies de Darwin, donde se genera soluciones a partir de otras conservando algunas características de estas de acuerdo al grado de mutación que se quiere logras en cada iteración, las mutación son realizadas de forma aleatoria, lo que resuelve la problemática de óptimos locales presentes en algunas soluciones.

\subsection{Métodos de búsqueda}

Son métodos que funcionan basándose en el hecho de que ya existe una solución inicial a la que le realizan modificaciones para mejorar y así obtener una solución cerca al optimo global del problema. Cuando se aplican estos métodos se corre el riesgo de llegar una solución de un óptimo local sin poder salir de ahí, pero los métodos desarrollados por lo general se centran en cómo salir del óptimo, para ello pueden retornar a una solución inicial diferente y volver a comenzar. (Multi start), hacer una variación de la estructura de entornos. (Metaheurística de búsqueda de entornos variables) y por último realizar movimientos que empeoren la solución para salir del óptimo local (Simulated nnealing y Búsqueda Tabú).

\section{- Búsqueda Tabú}

Es un algoritmo fundamentado en el concepto de movimientos prohibidos para la solución que va a ser mejorada, permitiendo navegar en un campo de posibles soluciones más amplio sin tener en cuenta las que ya se han encontrado, permitiendo establecer una "memoria" a corto y largo plazo, por lo que se considera una búsqueda inteligente, ya que aprende a medida que se realizan las iteraciones. Normalmente, opera con técnicas de diversificación aleatoria para salir de óptimos locales [14].

\section{PLANTEAMIENTO DEL PROBLEMA}

De acuerdo a los modelos matemáticos empleados en los sistemas de distribución y recolección de bienes o servicios, se considera la recolección de AU en Pereira como un CVRP (Capacited Vechicule Routing Problem) problema de ruteo de vehículos capacitado. El problema consiste en tener una flota de $\mathrm{M}$ vehículos con una capacidad homogénea y cada uno de ellos corresponde a una ruta de un vehículo con costo mínimo, donde el deposito es el punto de partida y de llegada, cada cliente es visitado una vez por un ciclo, la demanda de los nodos no excede la capacidad del vehículo, y se define el costo total como la suma de todos los arcos que pertenecen al ciclo.

Para la creación de la red de logística inversa se determinaron y ubicaron los 96 puntos generadores y el centro de acopio de AU en la ciudad de Pereira. También se construyó la matriz de costos de transporte (97 $x$ 97) entre todos los nodos que conforman la red. Se determinó la cantidad de galones de AU generados en cada uno de los 96 nodos. Para la recolección del residuo se cuenta con una flota de camiones homogénea con capacidad para transportar 3329 galones del desecho.

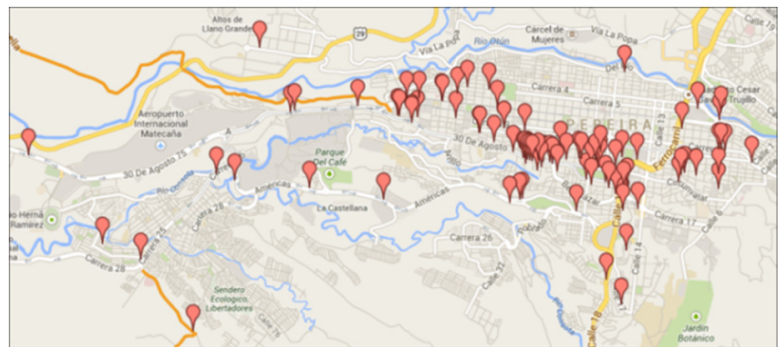

Figura 3. Mapa de puntos generadores de aceite usado en Pereira.

\section{FORMULACIÓN MATEMÁTICA}

El modelo de programación lineal entera (PLE) para CVRP, en el caso de la recolección de aceite usado en la ciudad de Pereira, puede definirse en un grafo $G=(V, A)$, donde $V=\{0,1,2,3, \ldots, n\}$ es un conjunto de $n=97$ nodos y $A$ representa el conjunto de $\operatorname{arcos}(i, j)$ que conectan dichos nodos. Asociado a cada arco del grafo existe una matriz $C=$ $\left(C_{i j}\right)$ de tamaño $n x n$ (97x97), con $C_{i j} \neq C_{j i}$. D es un arreglo de la forma $D_{i}$ que especifica la información de demanda de cada nodo.

El objetivo del problema consiste en encontrar un conjunto de rutas con el menor costo posible, que le permita a los vehículos $m$ recolectar el aceite generado por sus clientes. Cada uno de los vehículos $m$ cuenta con una capacidad máxima $P_{k}=3329$ galones, la cual es homogénea y no puede ser superada por la demanda de los clientes, que en total es de 11852 galones, lo que significa que un solo camión no puede recolectar todo el aceite, es necesario insertar varios a la red, permitiendo así, descartar que el problema del aceite vehicular usado sea un TSP (Traveling Salesman problem) problema del agente viajero, y se ajuste a las características del CVRP.

El modelo matemático del problema CVRP plantea que cada vehículo de la red inicie y finalice su recorrido en el depósito, que corresponde al nodo 0 (cero), todos los clientes deben ser atendidos una sola vez por un solo vehículo, y la demanda de cada cliente no puede ser fraccionada, o sea que la carga de un cliente es transportada completamente por un único vehículo, el que lo visita.

A continuación se presentan los componentes del modelo matemático del CVRP para la recolección de AU en Pereira:

Los índices del modelo son:

$i=$ nodo de partida $i(0,1,2, \ldots, 96)$

$j=$ nodo de llegada $j(1,2, \ldots, 96)$

$k=f \operatorname{lota}(1,2, \ldots, m)$ 
Los parámetros del problema son:

$C_{i j}=$ Costo de transporte del nodo $i$ al nodo $j$ (anexo 2 )

$D_{i}=$ Demanda en el nodo

$P_{k}=$ Capacidad del recursok

$n=$ Cantidad de puntos generadores de $A U$ (nodos)

Variable auxiliar:

$S=$ Demanda máxima que satisface un vehículo

La variable de decisión queda definida de la forma siguiente:

$$
X_{i j k}=\left\{\begin{array}{c}
1, \text { si el arco }(i, j) \text { es visitado por } k \\
0, \text { de lo contrario }
\end{array}\right\}
$$

$m=$ vehiculos necesarios para satisfacer la demanda

$$
\{m \geq 0 \text {, entero }\}
$$

Función Objetivo:

$$
\operatorname{Min} Z=\sum_{i=0}^{n} \sum_{j=0}^{n} \sum_{k=1}^{m} C_{i j} x_{i j k}
$$

La expresión (2) describe la función objetivo del problema, la cual consiste en minimizar la sumatoria de los costos asociadas a cada arco $(i, j)$ visitado por $k$.

Sujeto a:

$$
\begin{array}{lc}
\sum_{k=1}^{m} \sum_{j=1}^{n} X_{0 j k}=m & m \geq 0 \\
\sum_{k=1}^{m} \sum_{j=0}^{n} X_{i j k}=1 & \forall i \in[1, n] \\
\sum_{j=1}^{n} X_{0 j k}=\sum_{I=1}^{n} X_{0 J K} & \forall k \in[1, m] \\
\sum_{i=0}^{n} \sum_{j=0}^{n} D_{i} X_{I J K} \leq P_{k} & \forall k \in[1, m] \\
\sum_{\substack{i=1 \\
i \in S}} \sum_{j=1} X_{i j k} \leq|S|-1, \forall S \subseteq(V-\{0\}),|S| \geq 2, \\
\quad K \in(1, m), \forall(i, j) \in\{1, n\} \\
X_{i j} \in\{0,1\}, \forall(i, j) \in A & \forall k \in[1, m] \\
1 \leq k \leq m &
\end{array}
$$

La restricción (3) indica que del centro de acopio parten $m$ vehículos, la (4) y (5) garantizan que solo un vehículo visite y abandone cada cliente, la restricción (6) determina que la demanda total de los puntos generadores visitados por un camión no puede superar la capacidad máxima del vehículo $P_{k}$, este tipo de problema es conocido como Bin Packing Problem (BPP). La (7) establece la inexistencia de sub-tours, mientras que la (8) y (9) indica los valores admisibles para las variables.

\section{METODOLOGÍA DE SOLUCIÓN}

La solución del CVRP en la recolección de aceite vehicular usado en Pereira, es un problema de naturaleza NP-Hard y de optimización combinatoria compleja, que no puede ser resuelto con técnicas exactas por el gran tamaño (número de nodos o clientes) y el tiempo que tarda en encontrar el óptimo, ya que el tiempo de cálculo incrementa con rapidez a medida que los nodos $n$ aumentan y para el diseño de la red se cuenta con 96 puntos generadores de AU. Pero, si se pueden implementar otras técnicas que permiten obtener soluciones aproximadas en un tiempo razonable y sirven para la toma de decisiones, como son las heurísticas. Dentro de las cuales se pueden encontrar diferentes métodos adecuados para la solución del CVRP presentado anteriormente, que permiten construir una red de recolección con diferentes rutas que permitan satisfacer la demanda total de los clientes. [15]

Para resolver el problema se ha seleccionado una heurística de dos fases que consiste en rutear primero y agrupar después o route-first and cluster-second, la cual se divide en dos instancias, en la primera permite crear un gran ruta, usando una heurística constructiva como es el método de ahorro (Clarke and Wright), y en la segunda, de acuerdo a una restricción de capacidad en los camiones se asigna a cada vehículo una ruta con una cantidad de clientes determinada.

Ambos algoritmos son programados y solucionados en Matlab, un software que permite solucionar los algoritmos para encontrar las rutas con el costo mínimo y la asignación de cada una de ellas a los vehículos con capacidad limitada, garantizando la recolección del 100\% de aceite generado en los puntos de acopio en la ciudad de Pereira.

\section{Rutear primero o route-first}

En esta primera fase se emplea el método de ahorro o savings method de Clarke y Wright el cual genera una ruta donde se incluyen todos los nodos como si fueran a ser visitados por un solo vehículo sin tener en cuenta las restricciones de capacidad del problema.

Después de construida la información, se implementa el algoritmo del ahorro [16] para solucionar el VRP, que representa la primera fase del método, porque es una heurística de construcción y optimización que consiste en combinar los nodos de forma tal que al estar todos en una sola ruta (Gran ruta $\mathrm{R}$ ) se generen ahorros en los costos.

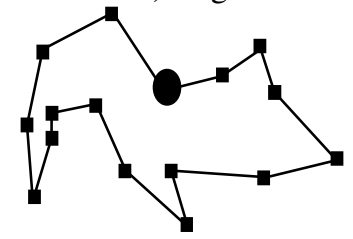

Figura 4. Gran ruta R (primera fase)

Además pertenece a las heurísticas clásicas para la solución de VRP y sus variantes que ofrecen una solución en una corta cantidad de tiempo y son fáciles de implementar, el algoritmo 
encuentra soluciones aproximadas de buena calidad, y también ha sido muy utilizado en aplicaciones prácticas, lo que significa que es un modelo digno de imitación en la solución de estos problemas.El algoritmo del ahorro en esta primera fase utiliza todos los nodos para encontrar una ruta $R=(0, i, \ldots, j, 0)$, como si se utilizara un solo vehículo para recórrela. El proceso empieza con una solución donde el camión parte del depósito y visita un solo cliente, después calcula el ahorro en distancias o costos para todas las combinaciones posibles.

Después de los cálculos se selecciona la combinación con el ahorro $S_{i j}$ mayor y se unen los nodos hasta tener la ruta. Por ejemplo, se tiene un depósito y tres nodos, cada arco formado entre los nodos y el depósito tienen unos costos asociados, como se muestra en la ilustración 6 , para determinar la ruta se calculan $\operatorname{los} S_{i j}$ asociados a cada pareja de nodos de la siguiente forma:

$S_{12}=S_{21}=6+5-8=3$

$S_{13}=S_{31}=6+9-2=13$

$S_{23}=S_{32}=5+9-3=11$

Luego se selecciona la pareja que más ahorro genera, en este caso es ir del nodo 1 al 2 o viceversa, luego esta de ir del nodo 2 al 3 o al contrario y por ultimo ir del nodo 1 al 3 o inversamente. Después de seleccionar que nodos se insertan de acuerdo al de mayor ahorro generado se tiene que la ruta es: 0-1-3-2-0 ó 0-2-3-1-0 con un costo mínimo total de 16, ya que los costos son iguales tanto de ida como de regreso, se puede iniciar en el nodo 1 o 2 y el resultado final será el mismo, lo anterior se puede ver en la figura 6 :
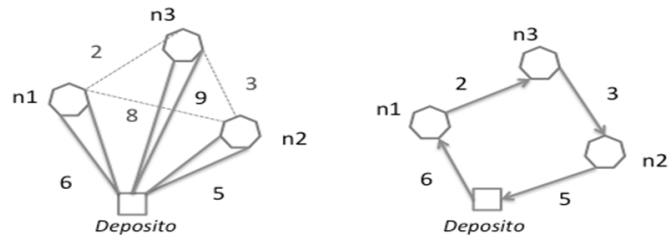

Figura 6. Ejemplo método del ahorro.

\section{Agrupar después o cluster-second}

En esta fase se realiza la creación de los clusters a través del algoritmo de agrupamiento o Clustering, porque en el CVRP, para la recolección de aceite usado en Pereira, los camiones seleccionados tienen una capacidad limitada de 3329 Galones y la demanda de todos los nodos no puede ser satisfecha por un solo vehículo, así que es necesario realizar una asignación de nodos a un número determinado de vehículos sin exceder la capacidad de los mismos.

El método asume esta segunda parte como un problema de empaquetamiento o embalaje BPP (Bin Packing Problem), el cual consiste en empacar un conjunto de objetos, en el caso del aceite usado es una cantidad en galones, y será transportado en varias cisternas o vehículos tipo cisterna de forma que el volumen máximo permitido en ellos no vaya a ser excedido.

El objetivo principal del problema consiste en minimizar el número total de vehículos y el costo por la utilización de ellos en la recolección del residuo, para ello el algoritmo se desarrolla bajo una única restricción de capacidad, dividiendo la gran ruta $\mathrm{R}$ en sub-rutas que terminan hasta que la capacidad del camión se cumpla o no se exceda, sin fraccionar las demandas de los clientes.

En el ejemplo de la figura 7, la gran ruta $R$ se subdivide en 3 sub-rutas, donde cada una de estas no puede superar la capacidad del camión recolector y deben ser recorridas por su respectivo vehículo recolector, por lo tanto se necesitan 3 camiones para satisfacer la demanda de la red en su totalidad.

Para el caso de la recolección de aceite usado, los 96 puntos generadores fueron agrupados considerando solo la restricción de capacidad en los vehículos, ya que la optimización de los costos y las distancias estaban determinadas en la fase anterior, lo que permitió realizar una agrupación siguiendo el orden de la ruta determinada por la gran ruta $R$.

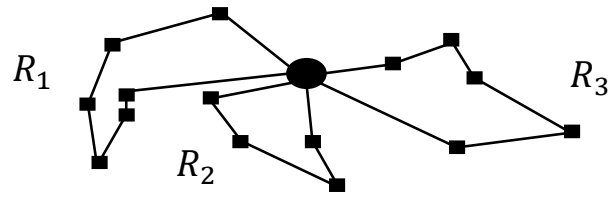

Figura 7. Sub-rutas $R_{i}$ (clusters)

Los 96 puntos se agruparan en 4 clusters, donde cada uno será atendido por un respectivo camión. Los camiones tienen capacidad limitada y la demanda total de estos no puede superar dicha capacidad. Cada clúster inicia y finaliza el recorrido en el depósito central.

Para encontrar los clusters, primero se debe definir la capacidad de los camiones recolectores que se utilizaran, en segundo lugar tener la ruta que se obtuvo en la fase 1 a través de la solución al problema de ruteamiento mediante el algoritmo de ahorro y conocer la demanda en todos los nodos.

\section{RESULTADOS}

Para determinar el mínimo costo se seleccionan dos tipos de camiones, ambos con diferente capacidad y costos de transporte, con la finalidad de poder seleccionar el mejor vehículo para el transporte del desecho. Se evaluaron y analizaron los resultados obtenidos con cada uno de los dos camiones y el mejor resultado se obtuvo utilizando el camión Dong Feng.

\section{Resultados utilizando el camión Dong Feng}

La marca del vehículo es DONG FENG y tiene una capacidad de almacenamiento de $3329 \mathrm{Gal}$. Al correr el modelo bajo esta condición, la red de logística inversa necesitará 4 camiones cisterna para recolectar la cantidad de lubricante usado 
producido por los puntos pertenecientes a la red. Por lo tanto, la flota estará compuesta por 4 vehículos recolectores y cada uno cubrirá una ruta específica. Las rutas asignadas a cada camión son:

- $\quad$ RutaA

\begin{tabular}{|c|c|c|c|}
\hline Camión & $\begin{array}{c}\text { Puntos } \\
\text { visitados }\end{array}$ & $\begin{array}{c}\text { Costo de la } \\
\text { ruta }\end{array}$ & $\begin{array}{c}\text { AU recolectado } \\
\text { (Gal) }\end{array}$ \\
\hline DONG FENG 1 & 21 & $\$ 35864,01$ & 3046 \\
\hline
\end{tabular}

Ruta A

\begin{tabular}{|l|c|c|c|c|c|c|c|c|c|c|c|}
\hline 0 & 96 & 95 & 92 & 91 & 88 & 87 & 84 & 83 & 80 & 79 & 76 \\
\hline 75 & 72 & 71 & 68 & 67 & 64 & 63 & 60 & 59 & 56 & 0 \\
\hline
\end{tabular}

Tabla 1. Ruta A

En esta ruta, el costo por galón recolectado del residuo es $11,77 \$ /$ Gal y el uso del tanque cisterna es del $91 \%$.

- $\quad$ Ruta B

\begin{tabular}{|c|c|c|c|c|c|c|c|c|c|c|c|c|}
\hline \multicolumn{4}{|c|}{ Camión } & \multicolumn{2}{|c|}{$\begin{array}{c}\text { Puntos } \\
\text { visitados }\end{array}$} & \multicolumn{3}{|c|}{$\begin{array}{l}\text { Costo de } \\
\text { la ruta }\end{array}$} & \multicolumn{4}{|c|}{$\begin{array}{c}\text { AU recolectado } \\
(\mathrm{Gal})\end{array}$} \\
\hline \multicolumn{4}{|c|}{ DONG FENG 2} & \multicolumn{2}{|c|}{46} & \multicolumn{3}{|c|}{$\$ 111178,89$} & \multicolumn{4}{|c|}{3283,5} \\
\hline \multicolumn{13}{|c|}{ Ruta B } \\
\hline 0 & 55 & 52 & 51 & 48 & 47 & 44 & 43 & 40 & & & 36 & 35 \\
\hline 32 & 31 & 28 & 27 & 24 & 23 & 20 & 19 & 16 & & & 12 & 11 \\
\hline 8 & 7 & 4 & 3 & 1 & 2 & 5 & 6 & 9 & & & 13 & 14 \\
\hline 17 & 18 & 21 & 22 & 25 & 26 & 29 & 30 & 33 & & & 37 & 0 \\
\hline
\end{tabular}

Tabla 2. Ruta B

El costo por galón recolectado del residuo es 33,86 \$/Gal y el uso del tanque cisterna es del $99 \%$.

\section{- $\quad$ Ruta C}

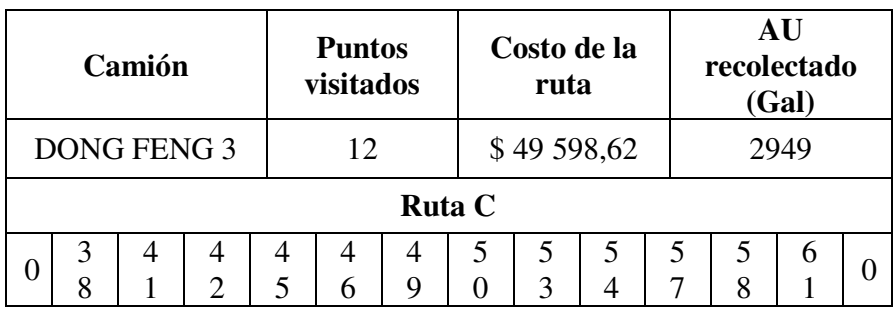

Tabla 3. Ruta C

El costo por galón recolectado del residuo es $16,82 \$ /$ Gal y el uso del tanque cisterna es del $88 \%$.

- $\quad$ Ruta D

\begin{tabular}{|c|c|c|c|c|c|c|c|c|c|c|c|}
\hline \multicolumn{2}{|c|}{ Camión } & $\begin{array}{c}\text { Puntos } \\
\text { visitados }\end{array}$ & $\begin{array}{c}\text { Costo de } \\
\text { la ruta }\end{array}$ & $\begin{array}{c}\text { AU recolectado } \\
\text { (Gal) }\end{array}$ \\
\hline DONG FENG 4 & 17 & $\$ 28284,9$ & \multicolumn{2}{|c|}{2543,5} \\
\hline \multicolumn{8}{|c|}{ Ruta D } \\
\hline 0 & 62 & 65 & 66 & 69 & 70 & 73 & 74 & 77 & 78 & 81 & 82 \\
\hline
\end{tabular}

\begin{tabular}{|l|l|l|l|l|l|l|}
\hline 85 & 86 & 89 & 90 & 93 & 94 & 0 \\
\hline
\end{tabular}

Tabla 4. Ruta D

El costo por galón recolectado del residuo es 11,12 \$/Gal y el uso del tanque cisterna es del $76 \%$.

\begin{tabular}{|c|c|c|c|}
\hline $\begin{array}{c}\text { Total } \\
\text { camiones } \\
\text { utilizados }\end{array}$ & $\begin{array}{c}\text { Puntos } \\
\text { visitados }\end{array}$ & $\begin{array}{c}\text { Costo total } \\
\text { de las rutas }\end{array}$ & $\begin{array}{c}\text { AU recolectado } \\
\text { (Gal) }\end{array}$ \\
\hline $\begin{array}{c}4 \text { camiones } \\
\text { DONG FENG }\end{array}$ & 96 & $\$ 224926,42$ & 11822 \\
\hline
\end{tabular}

Tabla 5. Resumen resultados rutas camión Dong Feng

Utilizando camiones DONG FENG para transportar el residuo, el uso promedio de las cisternas es del 88,5\% y el costo es menor en comparación con el otro escenario (camión FAW). El costo total de la ruta es de \$ 224926,42 y el costo por galón del residuo transportado es de 19,03 \$/Gal. Se deben comprar 4 camiones de este tipo para cubrir la demanda. Bajo este escenario se diseñará la red de logística inversa para recolectar AU en Pereira.

2. Diseño de la red de logística inversa para recolectar aceite vehicular usado

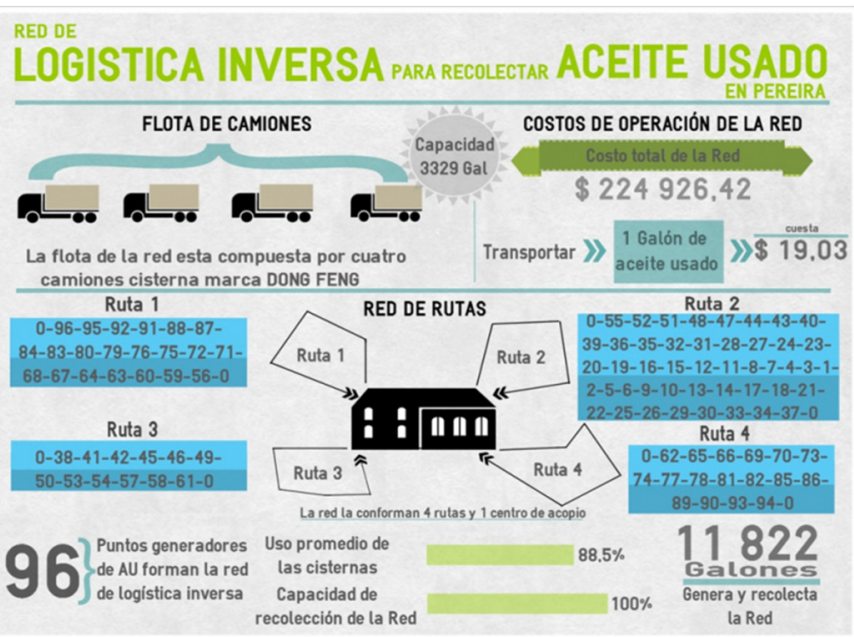

Figura 9. Red de logística inversa para recolectar AU en Pereira.

La figura 9, muestra cómo se aplicó el resultado arrojado por el modelo para diseñar la red de logística inversa. La red se compone de cuatro rutas, cada una atendida por un camión que hace parte de una flota de 4 vehículos que satisface la demanda del sistema. Las cisternas de los camiones tienen una capacidad de almacenamiento de 3329 galones. Cada ruta inicia el recorrido en el centro de acopio que está ubicado en la calle 20 entre carrera 12 y 13 , y regresa a este mismo punto con el lubricante usado que recolecto. El costo de operación del sistema de recuperación es \$ 224 926,42. Los 96 puntos generadores de AU que conforma la red de logística inversa producen 11822 galones del residuo y la red está en capacidad de recolectar el $100 \%$ del AU generado. La red de 
logística inversa tiene una capacidad de recuperación de 13 316 galones de AU, tiene una capacidad mayor a la generada por los 96 puntos.

\section{CONCLUSIONES}

- $\quad$ Se ubicaron 96 puntos que producen aceite usado en la ciudad de Pereira y se localizó el centro de almacenamiento del residuo en la calle 20 entre carrera 12 y 13 , debido a que los costos de transporte desde los nodos hasta este punto son menores.

- De acuerdo a las características y contexto de la recolección de aceite usado, se determinó que el problema de ruteamiento se ajustaba a un CVRP (Capacited Vehicle Routing Problem), porque los camiones cisterna empleados para la recolección tenían capacidad limitada y constante.

- Se implementó una heurística de dos fases para resolver este problema. Esta consiste en rutear primero y luego agrupar (Route-first and Clústersecond). Para resolver la primera fase se utilizó el Algoritmo de Ahorro y para la segunda fase el algoritmo para conformar los clúster. A través de esta metodología se resolvió el CVRP y se diseñó una red de logística inversa que la conforma un centro de acopio, 4 camiones, 4 rutas y 96 puntos generadores.

- Se determinó que la red de logística inversa diseñada, tiene una capacidad de recolección de 13316 galones de aceite vehicular usado. Los 96 puntos generadores que la conforman producen 11822 galones, por lo tanto solo se está utilizando el $89 \%$ de la capacidad de la red.

\section{REFERENCIAS}

[1] Monroy, Néstor; Ahumada, Claudia. "Logística Reversa: Retos para la Ingeniería Industrial”. En: Revista de ingeniería facultad de ingeniería Universidad de los Andes. Noviembre 2006. Vol. 23, pág. 23-33

[2] Ortega Mier, Miguel Ángel. Utilización de métodos cuantitativos para el análisis de problemas de localización en logística inversa. Tesis doctoral. Universidad Politécnica de Madrid. Madrid, España, 2008.

[3] Cruz, R. y Ertel, J. (2009). Reverse logistics network design for the collection of End-of-Life Vehicles in Mexico. En: European Journal of Operational Research, Vol. 196, pág. 930-939.
[4] Damon Gulczynski, Bruce L. Golden, Edward A. Wasil. The multi-depot split delivery vehicle routing problem: An integer programming-based heuristic, new test problems, and computational results. En: Computers \& Industrial Engeniering. 2011. Vol. 61 pág. 794-804.

[5] Li, F., Golden, B., \& Wasil, E. A record-to-record travel algorithm for solving the heterogeneous fleet vehicle routing problem. En: Computers \& Operations. 2007. Research 34, pág. $2734-2742$.

[6] Cordeau, J.-F., Laporte, G., \& Mercier, A. A unified tabu search heuristic for vehicle routing problems with time windows. En: Journal of the Operational Research Society. 2001. Vol 52, pág. 928-936.

[7] Networking Emerging Optimization Research Group. Universidad de Málaga, España. Branch and Bound. Recuperado el 10 de abril de 2014, del sitio web NEO: http://neo.lcc.uma.es/vrp/solution-methods/branch-and-bound/

[8] Naddef, Denis; RINALDI, Giovanni. Algoritmos Rama y corte para el VRP capacitado. El problema de ruteo de vehículos, 2002, vol. 9, pág. 53-81.

[9] Toth, Paolo; Vigo, Daniele. Algoritmos y con destino a Rama para el VRP capacitado. El problema de ruteo de vehículos, 2002, vol. 9, pág. 29-51.

[10] Corona León, José Alejandro. Hiperheurísticas a través de programación genética para la resolución de problemas de vehículos. Tesis (Magister en ciencias en sistemas inteligentes. Instituto tecnológico y de estudios superiores de Monterrey. México. 2005.

[11] Beasley, J.: Route first - cluster second methods for vehicle routing. En: Omega. 1983. Vol. 11 pág. 403-408

[12] Olivera, Alfredo. Heurísticas para problemas de ruteo de vehículos. Montevideo, Uruguay. 2004, 63p. Universidad de la Republica. Facultad de ingeniería. Instituto de Computación.

[13] Villegas R., Juan Guillermo. Heurísticas GRASP hibridas para el problema de rutas de vehículos con restricciones de capacidad. Tesis (Magister en Matemáticas Aplicadas). Universidad EAFIT, Escuela de ciencias y humanidades, Departamento de ciencias Básicas. Medellín, Colombia. 2008

[14] Vasquez DELGADO, Francisco de asís. Escuela técnica superior de ingenieros, universidad de Sevilla. 2012. Implementación de un algoritmo basado en la Búsqueda Tabú para la resolución de un problema de ruteo de vehículos con ventana temporal de acceso. Capítulo 4 resolución del problema de ruteo de vehículos VRP pág. 27-39 
[15] Lüer, Armin; Benavente, Magdalena; Bustos, Jaime; Venegas, Bárbara. El problema de rutas de vehículos: extensiones y métodos de resolución, estado del arte. En: Workshop Internacional EIG 2009 (Universidad de la Frontera, Chile). Diciembre 2009.

[16] Lysgaard, Jens. Clarke \& Wright Algoritmo de Ahorros. Departamento de Ciencias de la Gestión y Logística, la Aarhus School of Business, 1997. 